\title{
Fuzzy Comprehensive Evaluation Model of Enterprise M\&A Risk Based on AHP $^{*}$ Jing $\mathrm{Ma}^{1, \mathrm{a}}$ and Xueqiu Zhan ${ }^{2}$
}

\author{
${ }^{1}$ School of Economic Management, Wuxi Institute of Technology, Wuxi 214121, China \\ ${ }^{2}$ Department of Basic Course, Wuxi Institute of Technology, Wuxi 214121, China \\ ajilinmajing@163.com
}

Keywords: M\&A; risk evaluation; AHP

\begin{abstract}
Enterprise Merges and Acquisitions (M\&A) achieves the target of rapidly expand and increase asset scale, promote industrial upgrading and optimize resources allocation within the community as well as improve scale economic value of enterprises by transfer and reconstruct existing resources in the economic structure. However, the identification, evaluation and control on risk of enterprises M\&A are of severe shortage. The paper firstly analyzed and evaluated on six kinds of general evaluation model about enterprise M\&A risk. The disadvantages of these models were also given. On this basis, fuzzy comprehensive evaluation model based on AHP was presented. The specific application of comprehensive evaluation model was also provided.
\end{abstract}

\section{Introduction}

M\&A is a very complicated system. There are many uncertain factors in the M\&A process that M\&A process is complex, participants in a wide range, technical operation, professional and strong, operating cycle is longer and organizational decision-making coordination and cooperation required to implement high, so the risks faced by acquisition company are particularly large [1-4]. Enterprise M\&A risk refers to possibility that can not achieved intended goals in M\&A process as well as impact of it on normal management and business. At present, in the enterprise M\&A practices, the evaluation on M\&A risk only based on experiences, knowledge, ability and intuition of individual entrepreneurs. The understanding of its risk is only in the initial stage. In the theoretical world, there have been four ideas about risk evaluation on investment projects. The first is probability laws that mark the probability when investment income less than a standard income as risk evaluation index. The second is variance method that regards mean-variance or standard deviation of investment income ratio of project as risk evaluation index. The third is mean-standard deviation method that is use the ratio of standard deviation to mean as risk evaluation index. The forth is mean square error-loss law to regard the product of variance expected loss as index. Currently, most evaluation methods on M\&A risk are evolved from the basis of these ideas. Aiming at problems of empirical M\&A risk evaluation, the paper presents a fuzzy comprehensive evaluation model based on AHP. The paper is organized as follows: section 2 introduces 6 kinds of general evaluation models; section 3 gives fuzzy comprehensive evaluation model based on AHP; section 4 provides specific application example and section 5 concludes our work.

\section{M\&A Fuzzy Comprehensive Evaluation Model Based on AHP}

The main steps of fuzzy comprehensive evaluation are as following:

(1) Establish index set $U=\left\{u_{1}, u_{2}, \cdots, u_{n}\right\}$, namely evaluation index system.

(2) Establish evaluation set $V=\left\{v_{1}, v_{2}, \cdots, v_{n}\right\}$, namely schema set for evaluation.

\footnotetext{
*This work is supported Project supported by:the Strengthening SME Costing Study Foundation of Wuxi Institute of Technology No. JS2012-02 
(3) Establish weight set $A=\left\{a_{1}, a_{2}, \cdots, a_{n}\right\}$, namely relative improtace of different index, where, $\sum_{i=1}^{n} a_{i}=1$ and $a_{i}>0$.

(4) Establish fuzzy membership matrix, namely combination of membership vector to evaluate elements

$$
R=\left[\begin{array}{cccc}
r_{11} & r_{12} & \cdots & r_{1 n} \\
r_{21} & r_{22} & \cdots & r_{2 n} \\
\vdots & \vdots & \ddots & \vdots \\
r_{m 1} & r_{m 2} & \cdots & r_{m n}
\end{array}\right]
$$

Where, $r_{i j}(i=1,2, \ldots, m ; j=1,2, \ldots, n)$ is membership of index $U_{k i}$ in sub-element layer to the $j$-th level evaluation $V_{j}$. The value of $r_{i j}$ can be determined with the method as follows: statistically collect expert score and obtain that there is $V_{i 1}$ review at $V_{1}$ level for index $U_{k i}, V_{i 2}$ reviews at $V_{2}$ level, .., $V_{i m}$ reviews at $V_{m}$ level. For $i=1,2, \ldots, m$, there is:

$$
r_{i j}=V_{i j} / \sum_{j=1}^{n} V_{i j}(j=1,2, \cdots, n) .
$$

(5) Fuzzy comprehensive evaluation. Perform fuzzy synthesis operation $B=A \cdot R$, and then $B$ is the comprehensive evaluation result. So we can determine the level of object belongs to based on maximum membership principle.

\section{Case Study}

Here is the case of A company to acquisition B company to illustrate the specific application of the above model.

(1) According to AHP theory, establish index system of M\&A risk evaluation [8. 9] as shown in Fig. 1.

(2) Establish evaluation set.

Divide M\&A risk into 5 levels as high, higher, medium, lower and low to achieve the goal of early warning, namely $V=\left\{v_{1}, v_{2}, v_{3}, v_{4}, v_{5}\right\}=\{$ high, higher, medium, lower, low $\}$.

(3) Determine weight of indexes

Using Delphi method, we can obtain determination matrix at the appropriate level of each index. So we can arrive at relative weights as shown in Table. 1, Table. 2, Table. 3 and Table. 4.

From the above we can know that C.I. $=0.0327$ and C.R. $=0.0565$ in Table 1 ; C.I. $=0.0347$ and C.R. $=0.0310$ in Table 2; C.I. $=0.0606$ and C.R. $=0.0673$ in Table 3; C.I. $=0.0079$ and C.R. $=0.01362$ in Table 4. It means the consistency test is passed and acceptable.

(4) Establish fuzzy membership matrix R as shown in Table 5. with rating result from statistical experts

(5) M\&A risk evaluation

Firstly perform fuzzy matrix computation on evaluation matrix $R_{i}$ of each second level index $U_{i k}$ to obtain membership vector $B_{i}$ as shown in Table 6 of first-level index $U_{i}$ on review set $V$, $B_{i}=A_{i} \cdot R_{i}=\left\{v_{i 1}, v_{i 2}, v_{i 3}, v_{i 4}, v_{i 5}\right\}$.

Then conduct fuzzy operation on $R$, we can obtain membership vector $B$ of evaluation object on review set $V, \quad B=A \cdot R=\left(a_{1}, a_{2}, a_{3}\right) \cdot\left(B_{1}, B_{2}, B_{3}\right)^{T}$. Its comprehensive evaluation result is

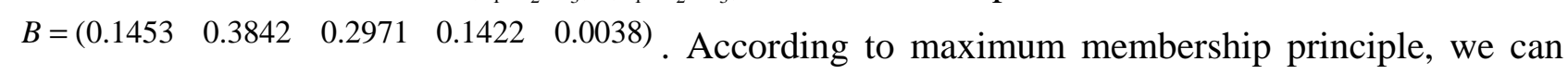
arrive at the following conclusion that the M\&A risk of the enterprise on target company is higher, which should be handled with care.

\section{Conclusion}

At present, the evaluation on M\&A risk only based on experiences, knowledge, ability and intuition of individual entrepreneurs in the enterprise M\&A practices. The understanding of its risk is 
only in the initial stage. To address the problem, the paper analyzed and evaluated on six kinds of general evaluation model about enterprise M\&A risk. The disadvantages of these models were given and fuzzy comprehensive evaluation model based on AHP was presented. The model application process and method were illustrated with specific case.

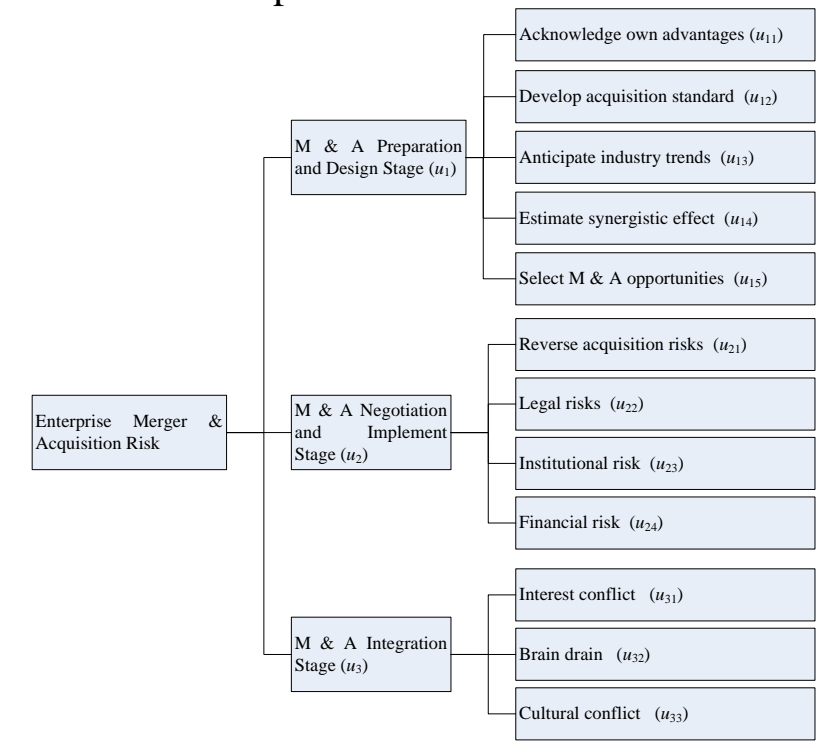

Figure 1. M\&A risk comprehensive index system.

TABLE 1. M\&A RISK DETERMINATION MATRIX IN STAGES

\begin{tabular}{|c|c|c|c|c|}
\hline $\boldsymbol{U}$ & $\boldsymbol{U}_{\mathbf{1}}$ & $\boldsymbol{U}_{\mathbf{2}}$ & $\boldsymbol{U}_{\mathbf{3}}$ & $\boldsymbol{a}_{\boldsymbol{i}}$ \\
\hline $\boldsymbol{U}_{\mathbf{1}}$ & 1 & $1 / 7$ & $1 / 5$ & 0.0738 \\
\hline $\boldsymbol{U}_{\mathbf{2}}$ & 7 & 1 & 3 & 0.6434 \\
\hline $\boldsymbol{U}_{\mathbf{3}}$ & 5 & $1 / 3$ & 1 & 0.2828 \\
\hline$\Sigma$ & 13 & 1.4762 & 4.2 & 1.0000 \\
\hline
\end{tabular}

TABLE 2. Risk DETERMinATION MATRIX IN PREPARATION AND DESIGN STAGE

\begin{tabular}{|c|c|c|c|c|c|c|}
\hline$U_{\mathbf{1}}$ & $\boldsymbol{U}_{\mathbf{1 1}}$ & $\boldsymbol{U}_{\mathbf{1 2}}$ & $\boldsymbol{U}_{\mathbf{1 3}}$ & $\boldsymbol{U}_{\mathbf{1 4}}$ & $\boldsymbol{U}_{\mathbf{1 5}}$ & $\boldsymbol{a}_{\mathbf{1 k}}$ \\
\hline $\boldsymbol{U}_{\mathbf{1 1}}$ & 1 & $1 / 3$ & $1 / 5$ & 2 & $1 / 2$ & 0.0848 \\
\hline $\boldsymbol{U}_{\mathbf{1 2}}$ & 3 & 1 & $1 / 3$ & 5 & 3 & 0.2454 \\
\hline $\boldsymbol{U}_{\mathbf{1 3}}$ & 5 & 3 & 1 & 7 & 5 & 0.4963 \\
\hline $\boldsymbol{U}_{\mathbf{1 4}}$ & $1 / 2$ & $1 / 5$ & $1 / 7$ & 1 & $1 / 3$ & 0.0500 \\
\hline $\boldsymbol{U}_{\mathbf{1 5}}$ & 2 & $1 / 3$ & $1 / 5$ & 3 & 1 & 0.1235 \\
\hline$\sum$ & 11.5 & 4.8667 & 1.8762 & 18 & 9.8333 & 1.0000 \\
\hline
\end{tabular}

TABLE 3. RISK DETERMINATION MATRIX IN NEGOTIATION AND IMPLEMENT STAGE

\begin{tabular}{|c|c|c|c|c|c|}
\hline $\boldsymbol{U}_{\mathbf{2}}$ & $\boldsymbol{U}_{\mathbf{2 1}}$ & $\boldsymbol{U}_{\mathbf{2 2}}$ & $\boldsymbol{U}_{\mathbf{2 3}}$ & $\boldsymbol{U}_{\mathbf{2 4}}$ & $\boldsymbol{a}_{\mathbf{2 k}}$ \\
\hline $\boldsymbol{U}_{\mathbf{2 1}}$ & 1 & 5 & 7 & 9 & 0.6545 \\
\hline $\boldsymbol{U}_{\mathbf{2 2}}$ & $1 / 5$ & 1 & 3 & 3 & 0.1849 \\
\hline $\boldsymbol{U}_{\mathbf{2 3}}$ & $1 / 7$ & $1 / 3$ & 1 & 3 & 0.1060 \\
\hline $\boldsymbol{U}_{\mathbf{2 4}}$ & $1 / 9$ & $1 / 3$ & $1 / 3$ & 1 & 0.0546 \\
\hline$\sum$ & 1.454 & 6.667 & 11.333 & 16 & 1.0000 \\
\hline
\end{tabular}

TABLE 4. RISK DETERMINATION MATRIX IN M\&A INTEGRATION STAGE

\begin{tabular}{|c|c|c|c|c|}
\hline $\boldsymbol{U}_{\mathbf{3}}$ & $\boldsymbol{U}_{\mathbf{3 1}}$ & $\boldsymbol{U}_{\mathbf{3 2}}$ & $\boldsymbol{U}_{\mathbf{3 3}}$ & $\boldsymbol{a}_{\mathbf{3 k}}$ \\
\hline $\boldsymbol{U}_{\mathbf{3 1}}$ & 1 & 1 & 5 & 0.4663 \\
\hline $\boldsymbol{U}_{\mathbf{3 2}}$ & 1 & 1 & 4 & 0.3830 \\
\hline
\end{tabular}




\begin{tabular}{|c|c|c|c|c|}
\hline $\boldsymbol{U}_{\mathbf{3 3}}$ & $1 / 5$ & $1 / 4$ & 1 & 0.1007 \\
\hline$\sum$ & 2.2 & 2.25 & 10 & 1.0000 \\
\hline
\end{tabular}

TABLE 5. M\&A RISK EVALUATION MATRIX

\begin{tabular}{|c|c|c|c|c|c|c|}
\hline \multirow{2}{*}{$\begin{array}{c}\boldsymbol{u}_{\boldsymbol{i}} \\
\left(\text { weight } \boldsymbol{a}_{\boldsymbol{i}} \text { ) }\right.\end{array}$} & \multicolumn{5}{|c|}{ Risk assessment value $\boldsymbol{r}_{\boldsymbol{i j}}$} \\
\cline { 2 - 7 } (weight $\boldsymbol{a}_{\boldsymbol{i k}}$ ) & High & Higher & Medium & Lower & Low \\
\hline \multirow{3}{*}{$\begin{array}{c}u_{1} \\
\left(a_{1}=0.0738\right)\end{array}$} & $u_{11}\left(a_{11}=0.0848\right)$ & 0.2 & 0.3 & 0.4 & 0.1 & 0.0 \\
\cline { 2 - 7 } & $u_{12}\left(a_{12}=0.2454\right)$ & 0.5 & 0.3 & 0.1 & 0.1 & 0.0 \\
\cline { 2 - 7 } & $u_{13}\left(a_{13}=0.4963\right)$ & 0.4 & 0.3 & 0.2 & 0.1 & 0.0 \\
\cline { 2 - 7 } & $u_{14}\left(a_{14}=0.0500\right)$ & 0.1 & 0.4 & 0.2 & 0.2 & 0.1 \\
\cline { 2 - 7 } & $u_{15}\left(a_{15}=0.1235\right)$ & 0.1 & 0.3 & 0.4 & 0.2 & 0.0 \\
\hline \multirow{3}{*}{$\left(a_{2}=0.6434\right)$} & $u_{21}\left(a_{21}=0.6545\right)$ & 0.1 & 0.5 & 0.3 & 0.1 & 0.0 \\
\cline { 2 - 7 } & $u_{22}\left(a_{22}=0.1849\right)$ & 0.1 & 0.3 & 0.4 & 0.2 & 0.0 \\
\cline { 2 - 7 } & $u_{23}\left(a_{23}=0.1060\right)$ & 0.0 & 0.1 & 0.4 & 0.5 & 0.0 \\
\cline { 2 - 7 } & $u_{24}\left(a_{24}=0.0546\right)$ & 0.0 & 0.1 & 0.3 & 0.5 & 0.1 \\
\hline \multirow{3}{*}{$\left(a_{3}=0.2828\right)$} & $u_{31}\left(a_{31}=0.4663\right)$ & 0.1 & 0.3 & 0.4 & 0.1 & 0.0 \\
\cline { 2 - 7 } & $u_{32}\left(a_{32}=0.3830\right)$ & 0.4 & 0.5 & 0.1 & 0.0 & 0.0 \\
\cline { 2 - 7 } & $u_{33}\left(a_{33}=0.1007\right)$ & 0.3 & 0.4 & 0.2 & 0.1 & 0.0 \\
\hline
\end{tabular}

TABLE 6. RISK DETERMINATION MATRIX IN EACH STAGE OF M\&A

\begin{tabular}{|c|c|c|c|c|c|}
\hline \multirow{2}{*}{$\boldsymbol{u}_{\boldsymbol{i}}$} & \multicolumn{5}{|c|}{ Risk assessment value $\boldsymbol{r}_{\boldsymbol{i} \boldsymbol{j}}$} \\
\cline { 2 - 6 } & High & Higher & Medium & Lower & Low \\
\hline$u_{1}$ & 0.3555 & 0.3050 & 0.2171 & 0.1174 & 0.0050 \\
\hline$u_{2}$ & 0.0839 & 0.3988 & 0.3291 & 0.1827 & 0.0055 \\
\hline$u_{3}$ & 0.2300 & 0.3717 & 0.2450 & 0.0567 & 0.0000 \\
\hline
\end{tabular}

\section{References}

[1] XU C. Y. and ZHOU W. Y.: Identification by Stages and Systematic Control of Business M\&A Risk, Science \& Technology Progress and Policy, vol. 6, 2004, pp. 62-64.

[2] HU Y. and REN J. H.: Measure and evaluation of the venture stopping coefficient for Merger \& Acquisition of enterprises, Journal of Wuhan University of Technology, vol. 25, 2003, pp. 89-91.

[3] CHU Y. P. and HU Y.: Risk Distinguishment of Merger and Acquisition of Enterprises and Frame Designment for Decision Supporting System, Technoeconomics \& Management Research, vol. 5, 2003, pp. 50-51.

[4] XU C. Y.: Structure, characteristic and operation of enterprise merger risk management system, East China Economic Management, vol. 19, 2005, pp. 65-67.

[5] DING M. Z. and LIU C. Z.: The fuzzy synthetic evaluating model on the personal credit of commercial bank based on AHP, Economy and Management, vol. 19, 2005, pp. 49-51.

[6] WU X. Y.: Fuzzy comprehensive evaluation on band crisis based on AHP, Science \& Technology Progress and Policy, vol. 3, 2005, pp. 126-127.

[7] ZHU B. X. and ZHU Z. H.: An Analysis of Risks in the Process of Merger and Acqusitions of Chinese Enterprises, Commercial Research, vol. 9, 2003, pp. 93-96.

[8] PAN J. and CHEN H. M.:An Empirical Analysis on M\&A Performance for Chinese Listed Companies, The Theory and Practice of Finance and Economics, vol. 26, 2005, pp. 69-73.

[9] TIAN B. P. and FENG Y. J.: Operating performance and risk evaluation of M\&A in listed companies in China, Journal of Harbin Institute of Technology(Social Sciences Edition), vol. 6, 2004, pp. 100-105. 